\title{
A Rotating Disk in the HH 111 Protostellar System
}

\author{
Chin-Fei Lee ${ }^{1}$
}

\begin{abstract}
The HH 111 protostellar system is a young Class I system with two sources, VLA 1 and VLA 2, at a distance of 400 pc. Previously, a flattened envelope has been seen in $\mathrm{C}^{18} \mathrm{O}$ to be in transition to a rotationally supported disk near the VLA 1 source. The follow-up study here is to confirm the rotationally supported disk at 2-3 times higher angular resolutions, at $\sim 0.3$ (or $120 \mathrm{AU}$ ) in $1.33 \mathrm{~mm}$ continuum, and $\sim 0.6$ (or $240 \mathrm{AU})$ in ${ }^{13} \mathrm{CO}(J=2-1)$ and ${ }^{12} \mathrm{CO}(J=2-1)$ emission obtained with the Submillimeter Array. The $1.33 \mathrm{~mm}$ continuum emission shows a resolved dusty disk associated with the VLA 1 source perpendicular to the jet axis, with a Gaussian deconvolved size of $\sim 240 \mathrm{AU}$. The ${ }^{13} \mathrm{CO}$ and ${ }^{12} \mathrm{CO}$ emissions toward the dusty disk show a Keplerian rotation, indicating that the dusty disk is rotationally supported. The density and temperature distributions in the disk derived from a simple disk model are found to be similar to those found in bright T-Tauri disks, suggesting that the disk can evolve into a T-Tauri disk in the late stage of star formation. In addition, a hint of a low-velocity molecular outflow is also seen in ${ }^{13} \mathrm{CO}$ and ${ }^{12} \mathrm{CO}$ coming out from the disk.
\end{abstract}

Subject headings: circumstellar matter - stars: formation — ISM: individual (HH 111)

\section{Introduction}

Stars are formed inside molecular cloud cores by means of gravitational collapse. The details of the process, however, are complicated by the presence of magnetic fields and angular momentum. In particular, for the stars to form, collapsing material has to lose most of the magnetic fields and angular momentum. As a result, in addition to infall (or collapse), rotation and outflow are also seen toward star-forming regions. In theory, a rotationally supported disk is expected to form inside a collapsing core around a protostar, from which

\footnotetext{
${ }^{1}$ Academia Sinica Institute of Astronomy and Astrophysics, P.O. Box 23-141, Taipei 106, Taiwan; cflee@asiaa.sinica.edu.tw
} 
part of the material is accreted by the protostar and part is ejected away. Observationally, however, when and how a rotationally supported disk is actually formed are still unclear, because of the lack of detailed kinematic studies inside the collapsing core.

This paper is a follow-up study of the HH 111 protostellar system, which is a well-studied Class I system deeply embedded in a compact molecular cloud core in the L1617 cloud of Orion at a distance of $400 \mathrm{pc}$. This system has a bolometric temperature $T_{\text {bol }} \sim 78 \mathrm{~K}$ and thus has just transitioned to the Class I phase from the Class 0 phase (Froebrich 2005). At the center of this system, there are two sources, VLA 1 and VLA 2, with a projected separation of $\sim 3^{\prime \prime}$ (1200 AU) (Reipurth et al. 1999). Previously, a flattened envelope has been seen in $\mathrm{C}^{18} \mathrm{O}$ around the VLA 1 source (Lee et al 2009, hereafter Paper I) and it seems to be in transition to a rotationally supported disk near the VLA 1 source (Lee et al 2010, hereafter Paper II). The follow-up study here is to confirm the rotationally supported disk at 2-3 times higher angular resolutions, at $\sim 0.3$ (or $120 \mathrm{AU}$ ) in $1.33 \mathrm{~mm}$ continuum, and $\sim 0$. 6 (or $240 \mathrm{AU}$ ) in ${ }^{13} \mathrm{CO}(J=2-1)$ and ${ }^{12} \mathrm{CO}(J=2-1)$ emission obtained with the Submillimeter Array (SMA)1 (Ho et al. 2004). This study also shows that the physical properties of the disk here in the early phase of star formation can be similar to those of the bright T-Tauri disks in the late phase of star formation (Andrews et al. 2009).

\section{Observations}

Observations toward the HH 111 system were carried out with the SMA, first on 2005 December 5 in the compact-north configuration (Paper I), then on 2010 January 24 in the extended configuration and on 2010 March 26 in the sub-compact configuration (Paper II), and then on 2011 Jan 01 in the very extended configuration. The baselines have projected lengths ranging from $\sim 10$ to $480 \mathrm{~m}$ onto the sky. The $230 \mathrm{GHz}$ band receivers were used to observe the ${ }^{12} \mathrm{CO}(J=2-1),{ }^{13} \mathrm{CO}(J=2-1), \mathrm{C}^{18} \mathrm{O}(J=2-1)$, and $\mathrm{SO}\left(N_{J}=5_{6}-4_{5}\right)$ lines simultaneously with the $1.33 \mathrm{~mm}$ continuum. The ${ }^{12} \mathrm{CO}$ and ${ }^{13} \mathrm{CO}$ lines are bright and thus are presented here to show the kinematics of the inner region and search for the rotationally supported disk around the VLA 1 source at high resolution. The velocity resolution in these lines is $\sim 0.28 \mathrm{~km} \mathrm{~s}^{-1}$ per channel, similar to that of $\mathrm{C}^{18} \mathrm{O}$ presented in Paper II. The visibility data were calibrated with the MIR package. The flux uncertainty was estimated to be $\sim 15 \%$. The calibrated visibility data in different configurations were combined and

\footnotetext{
${ }^{1}$ The Submillimeter Array is a joint project between the Smithsonian Astrophysical Observatory and the Academia Sinica Institute of Astronomy and Astrophysics, and is funded by the Smithsonian Institution and the Academia Sinica.
} 
then imaged with the MIRIAD package, as described in Paper II. With various different weightings, the synthesized beams can have a size from $\sim 0.6$ down to 0.3 . The rms noise levels are $\sim 0.036 \mathrm{Jy}^{\text {beam }}{ }^{-1}$ in the ${ }^{12} \mathrm{CO}$ channel maps with a beam of $0.58 \times 0.50,0.032 \mathrm{Jy}$ beam $^{-1}$ in the ${ }^{13} \mathrm{CO}$ channel maps with a beam of $0.66 \times 0.56$, and $0.9 \mathrm{mJy}^{\prime \prime}$ beam $^{-1}$ in the continuum map with a beam of $0.36 \times 0.31$. The velocities in the channel maps are LSR.

\section{Results}

As in Paper II, the results are presented in comparison to a mosaic image based on the Hubble Space Telescope (HST) NICMOS image ([FeII] $1.64 \mu \mathrm{m}+\mathrm{H}_{2}$ at $2.12 \mu \mathrm{m}+$ continuum) obtained by Reipurth et al. (1999), which shows the two sources VLA 1 and VLA 2 in the infrared, and the reflection nebulae that trace the illuminated outflow cavity walls. The two sources are located at $\alpha_{(2000)}=05^{\mathrm{h}} 51^{\mathrm{m}} 46^{\mathrm{s}} .254, \delta_{(2000)}=+02^{\circ} 48^{\prime} 29^{\prime \prime}$.65 (Rodríguez et al. 2008) and $\alpha_{(2000)}=05^{\mathrm{h}} 51^{\mathrm{m}} 46^{\mathrm{s}} .07, \delta_{(2000)}=+02^{\circ} 48^{\prime} 30^{\prime \prime} .76$, respectively (Reipurth et al. 1999; Rodríguez et al. 2008). The jet is bipolar with a western and an eastern component. It has a position angle (P.A.) of $\sim 97^{\circ}$ and an inclination angle of $\sim 10^{\circ}$, with the western component being blueshifted and tilted toward us (Reipurth. Raga, \& Heathcote 1992). The systemic velocity in this system is assumed to be $8.90 \pm 0.14 \mathrm{~km} \mathrm{~s}^{-1} \mathrm{LSR}$, as before. Throughout this paper, the velocity is relative to this systemic value.

\section{1. $\quad 1.33 \mathrm{~mm}$ Continuum Emission}

As shown in Figure 1a, the continuum emission associated with the VLA 1 source is now resolved into a disk-like structure perpendicular to the jet axis, with a Gaussian deconvolved (FWHM) size of $\sim 00^{\prime \prime} 6 \times 0^{\prime \prime} .3(\sim 240 \times 120 \mathrm{AU})$ and a P.A. of 7.6 ${ }^{\circ}$. As found in Paper II, this emission has a total flux of $\sim 285 \pm 40 \mathrm{mJy}$ and it is the thermal emission mainly from a dusty disk, with the inner part of which already seen with a deconvolved size of $\sim 0$."15 (60 AU) at $7 \mathrm{~mm}$ (Rodríguez et al. 2008). Note that the emission seems to show a little faint protrusion to the east along the jet axis. This faint protrusion seems to be seen in $3.6 \mathrm{~cm}$ and $7 \mathrm{~mm}$ as well, and it may trace either the material along the jet axis or a disk around a companion in a close binary system as suggested in Rodríguez et al. (2008). In addition, a faint protrusion is also seen extending to the southeast from the southern part of the disk. As in Paper II, faint emission is also detected around the VLA 2 source $(\sim 4 \sigma$ detection with $1 \sigma=0.9 \mathrm{mJy}_{\text {beam }}{ }^{-1}$ ), probably tracing a disk around it. Moreover, two more faint emission peaks DCN and DCS are seen at $3^{\prime \prime}$ in the north and $2^{\prime \prime}$ in the south respectively near the equatorial plane (P.A. $\sim 7^{\circ}$ ), probably tracing the density enhancement there. 


\subsection{Previous Results of Envelope and Outflow in $\mathrm{C}^{18} \mathrm{O}$}

Previously in the $\mathrm{C}^{18} \mathrm{O}$ observations (Paper II), a flattened envelope is seen perpendicular to the jet axis, extending to $\sim 7200 \mathrm{AU}\left(18^{\prime \prime}\right)$ out from the VLA 1 source. It is rotating, with the blueshifted emission in the north and the redshifted emission in the south. The outer part $\left(\sim 2000-7200 \mathrm{AU}\right.$, or $\left.\sim 5^{\prime \prime}-18^{\prime \prime}\right)$ seems to have a rotation that has constant specific angular momentum with $v_{\phi} \sim 3.90 d^{-1} \mathrm{~km} \mathrm{~s}^{-1}$, while the inner part $(\sim 60-2000 \mathrm{AU}$, or $\sim 0$.'15-5") seems to have a Keplerian rotation with $v_{\phi} \sim 1.75 d^{-0.5} \mathrm{~km} \mathrm{~s}^{-1}$, where $d$ is the radial distance from the source in the unit of arcsecond. Outflow is also seen in $\mathrm{C}^{18} \mathrm{O}$, with the blueshifted emission extending to the west and the redshifted emission extending to the east.

\subsection{Line Emission}

The ${ }^{13} \mathrm{CO}$ and ${ }^{12} \mathrm{CO}$ lines are detected toward the VLA 1 source upto $\sim 4.2 \mathrm{~km} \mathrm{~s}^{-1}$ and $10 \mathrm{~km} \mathrm{~s}^{-1}$, respectively, from the systemic velocity. In order to show how the envelope and disk structure changes with velocity, the line emission is divided into four velocity ranges: low $\left(0-2.5 \mathrm{~km} \mathrm{~s}^{-1}\right)$, medium $\left(2.5-4.2 \mathrm{~km} \mathrm{~s}^{-1}\right)$, high $\left(4.2-5.8 \mathrm{~km} \mathrm{~s}^{-1}\right)$, and very high $(5.8-10$ $\mathrm{km} \mathrm{s}^{-1}$ ) velocity ranges, on the redshifted and blueshifted sides.

In ${ }^{13} \mathrm{CO}$, at low velocity, the redshifted emission is detected mainly in the south and the blueshifted emission is mainly in the north of the VLA 1 source (Fig. 2a), consistent with a rotation motion about the source. As seen in $\mathrm{C}^{18} \mathrm{O}$, the emission is contaminated by the outflow emission, with the blueshifted emission also extending to the west and the redshifted emission also extending to the east. In addition, the blueshifted emission shows a V-shaped structure opening to the north, spatially coincident with the cavity walls traced by the reflection nebulae, suggesting that the envelope material is piling up on the cavity walls as the cavity walls expand into the envelope. A similar V-shaped structure is also seen opening to the south in the redshifted emission. At medium velocity, the ${ }^{13} \mathrm{CO}$ emission shrinks to the source, spatially coincident with the dusty disk (Fig. 2a and Fig. 1b for a zoom-in). The blueshifted emission peak is to the north and the redshifted emission peak is to the south in the equatorial plane, indicating that the motion there is highly dominated by the rotation about the VLA 1 source, as expected for a rotationally supported disk. The two peaks, however, are not symmetric about the source, with the northern peak at a distance of $\sim 0$. 25 and southern peak at $\sim 0.0^{\prime \prime}$. Two faint protrusions are also seen, one in the blue extending to the west from the northern part of the disk and one in the red extending to the east from the southern part, parallel to the jet axis. These structures are similar to those seen in the rotating molecular outflow in CB 26 (Launhardt et al. 2009), and thus may trace 
material outflowing from the disk.

In ${ }^{12} \mathrm{CO}$, at low velocity, the blueshifted emission is mainly to the north and the redshifted emission is mainly to the south, with V-shaped structures coincident with the cavity walls (Fig. 2k), similar to that seen in ${ }^{13} \mathrm{CO}$. At medium velocity, the blueshifted emission extends to the west from the northern part of the disk while the redshifted emission extends to both the west and east from the southern part of the disk (Fig. 2d and Fig. 11 for a zoomin). Note that the blueshifted emission and the redshifted emission beyond $\sim \pm 1^{\prime \prime}$ from the source are contaminated by the outflow shell emission, showing a cone-like structure with the tip pointing toward the source. The blueshifted emission also shows an E-W elongation at $\sim 3^{\prime \prime}$ in the north (Fig. 2d), with an unknown origin. At high velocity, the blueshifted and redshifted emissions extend out from the inner radii of the disk (Fig. 2e and Fig. 11d for a zoom-in). The emissions within $\sim 1^{\prime \prime}$ from the source are not much contaminated by the outflow shell emission, which is expected to be further away from the source at higher velocity. They extend to the west, first opening from the VLA 1 source and then bent to be aligned with the jet axis. They also extend slightly to the east. The blueshifted emission is mainly to the north of the jet axis and the redshifted emission is mainly to the south, with the velocity sense the same as that of the rotation in the disk, and thus may trace the material coming out from the disk. Note that, in the west, the blueshifted emission also extends slightly to the south. At very high velocity, only blueshifted emission is detected with a peak slightly away from the equatorial plane at $\sim 0$.'25 to the west (Fig. 2f and Fig. 17 for a zoom-in). This emission peak is spatially coincident with the blueshifted emission peak seen at high velocity in Figure 1d, suggesting that the emission there has a broad range of velocities from high to very high and may trace a shock interaction, either inside the disk or outside.

\subsubsection{Rotation motion near the VLA 1 source}

As seen in the last section, the inner part of the envelope can also be traced by ${ }^{13} \mathrm{CO}$ and ${ }^{12} \mathrm{CO}$, allowing us to check and confirm the Keplerian rotation law near the source seen in $\mathrm{C}^{18} \mathrm{O}$. Figure 3 shows the position-velocity (PV) diagrams of the envelope and disk in ${ }^{13} \mathrm{CO}$ and ${ }^{12} \mathrm{CO}$ cut along the equatorial plane perpendicular to the jet axis. In ${ }^{13} \mathrm{CO}$, the emission within $\pm 1 \mathrm{~km} \mathrm{~s}^{-1}$ from the systemic velocity is mainly resolved out and affected by the outflow emission, and thus should be excluded for studying the rotation law. As can been seen, the velocity structure beyond $\pm 1 \mathrm{~km} \mathrm{~s}^{-1}$ roughly follows the Keplerian rotation law seen in $\mathrm{C}^{18} \mathrm{O}$. In ${ }^{12} \mathrm{CO}$, the emission within $\pm 2 \mathrm{~km} \mathrm{~s}^{-1}$ from the systemic velocity is mainly resolved out and affected by the outflow emission, and thus should also be excluded 
for studying the rotation law. In addition, as seen in the emission map (Fig. 2d and e), the blueshifted emission is also detected at $\sim 2^{\prime \prime}$ to $3^{\prime \prime}$ in the north with a velocity ranging from -2 to $-6 \mathrm{~km} \mathrm{~s}^{-1}$ w.r.t. the systemic velocity. This emission is unclear, and it may trace some interaction in the envelope or something in the foreground or background, and thus should also be excluded. In this line, on the redshifted side, the emission is detected up to $\sim 6 \mathrm{~km} \mathrm{~s}^{-1}$, higher than that seen in ${ }^{13} \mathrm{CO}$, arising from the inner region as seen in Figure 11. The velocity structure also follows the Keplerian rotation law to that high velocity to the inner region. On the blueshifted side, the emission is detected up to $-10 \mathrm{~km} \mathrm{~s}^{-1}$ from the systemic, much higher than that on the redshifted side. However, as can be seen, the emission beyond $-5 \mathrm{~km} \mathrm{~s}^{-1}$ is localized with a broad range of velocities and it corresponds to the localized emission at $\sim 0$.'25 to the west as seen in the emission map (Fig. 18). Since this emission can trace a local shock interaction, the blueshifted emission should not be used to derive the rotation law. As a result, the rotation structure near the source seen in ${ }^{12} \mathrm{CO}$ is also consistent with that seen in $\mathrm{C}^{18} \mathrm{O}$.

\section{Model the disk continuum emission}

Here a simple disk model is used to fit the structure and intensity distribution of the continuum emission in order to derive the temperature and density distributions of the dusty disk. Note that, the emission that extends to the east along the jet axis and the emission that extends to the southeast from the southern part of the disk are not from the disk perpendicular to the jet axis and are thus excluded from the model.

For simplicity, the disk in the model is assumed to be flat with a constant thickness of $H$, an inner radius of $R_{\text {in }}$, and an outer radius of $R_{\text {out }}$. The disk is assumed to be perpendicular to the jet axis and thus have an inclination angle of $10^{\circ}$ to the line of sight, with the nearside tilted to the east (Reipurth, Raga, \& Heathcote 1992). It has a position angle of $7.6^{\circ}$ as given by the Gaussian source deconvolution earlier in Section 3.1. The temperature is assumed to be given by

$$
T=T_{0}\left(\frac{R}{R_{0}}\right)^{-q}
$$

where $R_{0}=0$ ".3 (120 AU), which is the radius (i.e., the half of the size) of the disk derived earlier from the Gaussian source deconvolution, $T_{0}$ is the temperature at $R_{0}$, and the powerlaw index $q$ is set to 0.5 , assumed to be similar to that found in T-Tauri disks in the late stage of star formation (Mundy et al. 1996; Andrews et al. 2009). In Paper I, using the dust opacity law

$$
\kappa_{\nu}=0.1\left(\frac{\nu}{10^{12} \mathrm{~Hz}}\right)^{\beta} \mathrm{cm}^{2} \mathrm{~g}^{-1}
$$


as given in Beckwith et al. (1990), the spectral energy distribution (SED) of the continuum source was found to be fitted with a dust temperature ranging from 41 to $64 \mathrm{~K}$ and $\beta \sim 0.9$. Thus, here $T_{0}$ is assumed to be $45 \mathrm{~K}$ and the dust mass opacity is assumed to be $0.026 \mathrm{~cm}^{2}$ $\mathrm{g}^{-1}$ at $\lambda=1.33 \mathrm{~mm}$ ( or $225 \mathrm{GHz}$ ). The number density of molecular hydrogen is assumed to be given by

$$
n=n_{0}\left(\frac{R}{R_{0}}\right)^{-p}
$$

with the power-law index $p$ assumed to be 1 . With a constant thickness, the disk has a surface density with a power-law index of 1 , similar to that found in the T-Tauri disks (Andrews et al. 2009). As can be seen in Figure 4, with the above assumptions, the model can fit the continuum emission reasonably well, with $H \sim 0$ ".3 (120 AU), $R_{\text {in }} \sim 0$.'03 (12 AU), $R_{\text {out }} \sim 0^{\prime \prime} 6$ (240 AU), and $n_{0} \sim 10^{9} \mathrm{~cm}^{-3}$. Here, $H$ turns out to be the same as the Gaussian source deconvolved size of the continuum emission in the minor axis. This is expected for a nearly edge-on disk, for which the size in the minor axis is mainly due to the thickness of the disk. On the other hand, $R_{\text {out }} \sim 2 R_{0}$ and thus is twice the disk radius derived from the Gaussian source deconvolution. Assuming that Helium has a number density $n_{\mathrm{He}}=0.2 n$, then the mean molecular weight with respect to the number of molecular hydrogen is 2.8 . Thus, the mass of the disk is found to be $0.14 M_{\odot}$, similar to the mean value found by fitting the SED (Paper II). The surface density in the disk is $\sim 7.2 \mathrm{~g} \mathrm{~cm}^{-2}$ at $120 \mathrm{AU}$ and $21.5 \mathrm{~g} \mathrm{~cm}^{-2}$ at $40 \mathrm{AU}$, similar to those found in the bright T-Tauri disks, e.g., GSS 39, in Ophiuchus, which also has a similar radius of $\sim 200 \mathrm{AU}$ and a same disk mass of $\sim 0.14 M_{\odot}$ (Andrews et al. 2009). Thus, the dusty disk here can be a very young protoplanetary disk that could evolve into a T-Tauri disk in the later stage of star formation.

\section{Discussion}

\subsection{A Rotationally supported Disk}

A rotationally supported disk has been seen in Taurus with an outer radius of $\sim 500 \mathrm{AU}$ in CO in the late (i.e., Class II or T Tauri) phase of star formation (see, e.g., Simon et al. 2000). Note that the disk could appear smaller in continuum (Andrews et al. 2009), because of the rapid decrease in the continuum intensity due to a power-law decrease of the density and temperature in the disk. Formation of such a disk must have started early in the Class I phase and even earlier in the Class 0 phase. HH 111, with a bolometric temperature of only $\sim 78 \mathrm{~K}$, is a very young Class I source, just transitioning from the Class 0 phase. The observations here show that such a rotationally supported disk can indeed form in such an early phase of star formation. In continuum, the disk is found to have a deconvolved 
radius of $\sim 120 \mathrm{AU}$ (with an outer radius of $240 \mathrm{AU}$ in the disk model), with the inner part of which already seen with a deconvolved radius of $\sim 30 \mathrm{AU}$ at $7 \mathrm{~mm}$ (Rodríguez et al. 2008). It is rotationally supported with a Keplerian rotation, as seen previously in $\mathrm{C}^{18} \mathrm{O}$ and now in ${ }^{13} \mathrm{CO}$ and ${ }^{12} \mathrm{CO}$, which implies a mass of $\sim 1.3 M_{\odot}$ for the central star (Paper II). Note that as discussed in Paper II, this stellar mass can account reasonably for the observed bolometric luminosity. The disk mass is $\sim 0.14 M_{\odot}$, which is $\sim 10 \%$ of the stellar mass. This disk mass is consistent with that implied for a Class 0 source by Jørgensen et al. (2009) and that implied for a young Class I source by Andrews \& Williams (2007). Since no rotationally supported disk has been confirmed in the Class 0 phase, the disk here could be the youngest rotating disk resolved to date. Moreover, as discussed earlier, the surface density and the radius of the disk here in $\mathrm{HH} 111$ are both similar to those of the bright resolved T-Tauri dusty disks in Andrews et al. (2009). Thus, the disk could evolve to a T-Tauri disk in the later stage of star formation as the accretion rate drops to $10^{-7} M_{\odot} \mathrm{yr}^{-1}$ from a few times $10^{-6} M_{\odot} \mathrm{yr}^{-1}$ (Paper II $)$.

As discussed in Paper II, the Keplerian rotation in this system can extend further out to the envelope to $\sim 2000 \mathrm{AU}\left(5^{\prime \prime}\right)$ from the source. At that large radius, the envelope seems to have some small amount of infall motion as well, and thus the rotation there could actually be sub-Keplerian. Therefore, the disk seems to be deeply embedded in a subKeplerian envelope with a radius of $\sim 2000$ AU. Observations at higher velocity and angular resolutions are really needed to differentiate the Keplerian motion from the sub-Keplerian motion in order to determine where the envelope is transitioning to a disk. Another way to determine the location of transition is to search for an accretion shock that is expected to occur at the transition. As discussed in Paper II, SO is found to trace a shock emission at $400 \mathrm{AU}\left(1^{\prime \prime}\right)$, and it may trace an accretion shock and thus the transition there. Further observations in other shock tracers are needed to check this possibility.

Rotationally supported disks have also been claimed in a few other Class I sources, e.g., IRAS $04302+2247$ (the Butterfly star, $T_{\text {bol }}=203 \mathrm{~K}$ ) (Wolf et al. 2008), L1489-IRS $\left(T_{\text {bol }}=238 \mathrm{~K}\right)\left(\right.$ Brinch et al. 2007), IRS $43\left(T_{\text {bol }}=310 \mathrm{~K}\right)($ Jørgensen et al. 2009), and Elias $29\left(T_{\text {bol }}=350 \mathrm{~K}\right)\left(\right.$ Lommen et al. 2008). These sources, with a much higher $T_{\text {bol }}$ than $\mathrm{HH}$ 111, are at much later stages than HH 111. Among these sources, L1489-IRS has been modeled in detail for the disk and envelope properties, and thus can be compared here with HH 111. This system was found to host a Keplerian disk with an outer radius of $\sim 200$ AU deeply embedded in a sub-Keplerian envelope with a radius $\sim 2000 \mathrm{AU}$ (Brinch et al. 2007), similar to the HH 111 system. The mass and the infall rate in the sub-Keplerian envelope are $\sim 0.093 M_{\odot}$ and $\sim 4.3 \times 10^{-6} M_{\odot} \mathrm{yr}^{-1}$, respectively (Brinch et al. 2007), also similar to those in HH 111, which are $\sim 0.13 M_{\odot}$ and $4.2 \times 10^{-6} M_{\odot} \mathrm{yr}^{-1}$, respectively (Paper II). The central star has a mass of $\sim 1.35 M_{\odot}$, also similar to that in $\mathrm{HH} 111$. However, this system has a 
bolometric luminosity of $\sim 3.7 L_{\odot}$, much smaller than that of $\mathrm{HH} 111$, which is $\sim 20 L_{\odot}$. The small bolometric luminosity suggests that the accretion rate in the disk must be much lower than that in $\mathrm{HH}$ 111, as supported by the less jet activity. This is probably because that the accretion rate decreases with the evolutionary stage. The disk mass was first found to be only $4 \times 10^{-3} M_{\odot}$ (Brinch et al. 2007) and later modified to be $0.018 M_{\odot}$ (Jørgensen et al. 2009), but it is still much smaller than that in HH 111. As argued in Jørgensen et al. (2009), however, it is not clear if this is because the disk mass really decreases with the evolutionary stage, or merely because the disk mass is underestimated more in the later stage due to a grain growth.

Rotationally supported disks have been seen in a few other much older sources, eg., HH 30 (late Class I) (Guilloteau et al. 2008) and IRAS 04158+2805 (Class II) (Andrews et al. 2008). In these older disks, a central hole is seen and it could be due to a tidal clearing by a possible binary system at the center or due to an outflow cavity. Since the HH 111 VLA 1 source itself is a close binary, a central hole is expected to have formed at the center of the disk near the source as well. Observations at higher angular resolution are needed to check this possibility.

\subsection{Material outflowing from the disk?}

As mentioned in Section [3.3, ${ }^{12} \mathrm{CO}$ and ${ }^{13} \mathrm{CO}$ emissions are seen near the source extending out from the disk to the east and west parallel to the jet axis. Could this emission structure trace the material coming out from the disk, as that seen in CB 26 (Launhardt et al. 2009), carrying away the angular momentum? To study this possibility, PV diagrams cut across the jet axis centered at 5 positions along the jet axis, from $-1^{\prime \prime} 2$ in the east to $1^{\prime \prime} 2$ in the west with a step of 0.6 (which is the size of the synthesized beam), are presented here in Figure 5 to investigate the kinematics of this emission structure. In these cuts, excluding the emission within $\pm 2 \mathrm{~km} \mathrm{~s}^{-1}$ from the systemic velocity, the blueshifted emission is in the north and redshifted in the south, as seen in the emission map in Figure 1 d, with a velocity sense the same as that of the rotation in the disk. Therefore, the emission could trace the material rotating around the jet axis and thus the material outflowing from the disk. In panels (c) and (d), the blueshifted emission is contaminated by the shock emission and should be excluded. From cut $\mathrm{A}$ in the east to cut $\mathrm{E}$ in the west, no clear systemic velocity shift is seen in the PV diagrams. Thus, if the emission really traces the material outflowing from the disk, the projected outflow velocity must be smaller than $1 \mathrm{~km} \mathrm{~s}^{-1}$. This is not impossible since (1) the outflowing material could have the same inclination as the jet and thus be close to the plane of the sky with an inclination angle of $\sim 10^{\circ}$, and (2) the 
outflowing velocity could be only a few $\mathrm{km} \mathrm{s}^{-1}$ in ${ }^{12} \mathrm{CO}$ (Launhardt et al. 2009). Further observations are needed to study this possibility.

\section{Conclusion}

The HH 111 protostellar system has been mapped at a resolution of $\sim 0^{\prime \prime} .3$ in $1.33 \mathrm{~mm}$ continuum, and 0.6 in ${ }^{13} \mathrm{CO}(J=2-1)$ and ${ }^{12} \mathrm{CO}(J=2-1)$. The $1.33 \mathrm{~mm}$ continuum emission shows a resolved dusty disk associated with the VLA 1 source perpendicular to the jet axis, with a Gaussian deconvolved size of $\sim 240 \mathrm{AU}$. The ${ }^{13} \mathrm{CO}$ and ${ }^{12} \mathrm{CO}$ emissions toward the dusty disk show a Keplerian rotation, indicating that the dusty disk is rotationally supported. The density and temperature distributions in the disk derived from a simple disk model are found to be similar to those found in bright T-Tauri disks. Thus, the disk can be a young protoplanetary disk that could evolve into a T-Tauri disk in the late stage of star formation. In addition, a hint of a low-velocity molecular outflow is also seen in ${ }^{13} \mathrm{CO}$ and ${ }^{12} \mathrm{CO}$ coming out from the disk.

I thank the SMA staff for their efforts in running and maintaining the array. I also thank the referee for the valuable comments.

\section{REFERENCES}

Andrews, S. M., Liu, M. C., Williams, J. P., \& Allers, K. N. 2008, ApJ, 685, 1039

Andrews, S. M., Wilner, D. J., Hughes, A. M., Qi, C., \& Dullemond, C. P. 2009, ApJ, 700, 1502

Andrews, S. M., \& Williams, J. P. 2007, ApJ, 671, 1800

Beckwith, S. V. W., Sargent, A. I., Chini, R. S., \& Guesten, R. 1990, AJ, 99, 924

Brinch, C., Crapsi, A., Hogerheijde, M. R., \& Jørgensen, J. K. 2007, A\&A, 461, 1037

Froebrich, D. 2005, ApJS, 156, 169

Guilloteau, S., Dutrey, A., Pety, J., \& Gueth, F. 2008, A\&A, 478, L31

Ho, P. T. P., Moran, J. M., \& Lo, K. Y. 2004, ApJ, 616, L1 
Jørgensen, J. K., van Dishoeck, E. F., Visser, R., Bourke, T. L., Wilner, D. J., Lommen, D., Hogerheijde, M. R., \& Myers, P. C. 2009, A\&A, 507, 861

Launhardt, R., et al. 2009, A\&A, 494, 147

Lee, C.-F., Mao, Y.-Y., \& Reipurth, B. 2009, ApJ, 694, 1395

Lee, C.-F. 2010, ApJ, 725, 712

Lommen, D., Jørgensen, J. K., van Dishoeck, E. F., \& Crapsi, A. 2008, A\&A, 481, 141

Mundy, L. G., et al. 1996, ApJ, 464, L169

Reipurth, B., Raga, A. C., \& Heathcote, S. 1992, ApJ, 392, 145

Reipurth, B., Yu, K. C., Rodríguez, L. F., Heathcote, S., \& Bally, J. 1999, A\&A, 352, L83

Rodríguez, L. F., Torrelles, J. M., Anglada, G., \& Reipurth, B. 2008, AJ, 136, 1852

Simon, M., Dutrey, A., \& Guilloteau, S. 2000, ApJ, 545, 1034

Wolf, S., Schegerer, A., Beuther, H., Padgett, D. L., \& Stapelfeldt, K. R. 2008, ApJ, 674, L101 

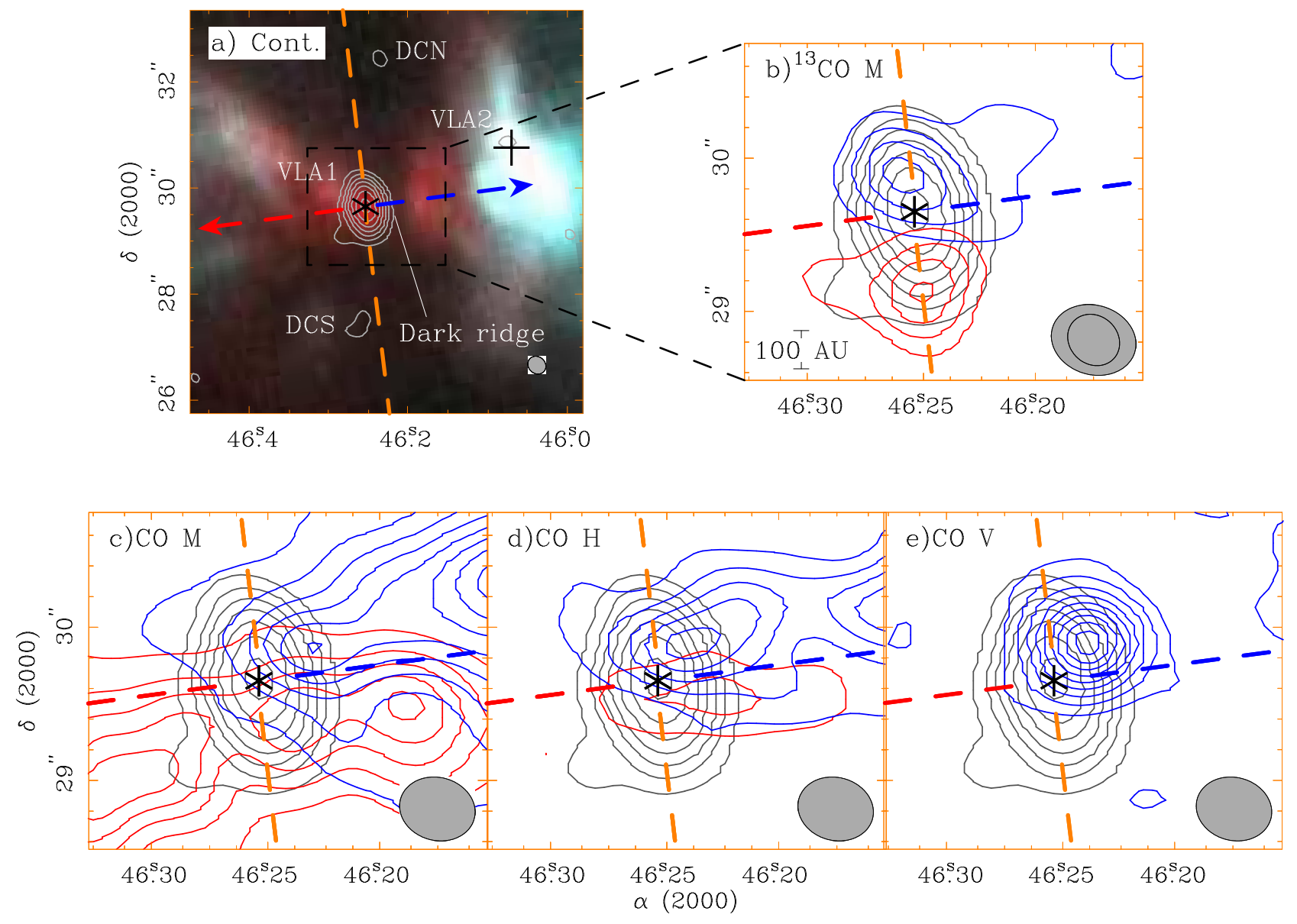

Fig. 1.- $1.33 \mathrm{~mm}$ continuum, ${ }^{13} \mathrm{CO}$, and ${ }^{12} \mathrm{CO}$ emission contours overplotted on the HST NICMOS image adopted from Reipurth et al. (1999). The asterisk and cross mark the positions of the VLA 1 and 2 sources, respectively. The blue and red arrows indicate the orientations of the blueshifted and redshifted components of the jet, respectively. The dashed line indicates the equatorial plane perpendicular to the jet axis. The synthesized beam has a size of $0.36 \times 0.31$ in continuum, $0.66 \times 0.56$ in ${ }^{13} \mathrm{CO}$, and $0 . .58 \times 0.50$ in ${ }^{12} \mathrm{CO}$. The gray contours are the continuum contours with levels of $4 \sigma\left(1-r^{n}\right) /(1-r)$, where $r=1.45$, $\mathrm{n}=1,2,3 .$. , and $\sigma=0.9 \mathrm{mJy}_{\text {beam }}{ }^{-1}$. (b) shows the redshifted (red contours) and blueshifted (blue contours) ${ }^{13} \mathrm{CO}$ emission at medium velocity. The contour levels start at $4 \sigma$ with a step of $3 \sigma$, where $\sigma=0.023 \mathrm{Jy}_{\text {beam }^{-1}}$. (c), (d), and (e) show the redshifted (red contours) and blueshifted (blue contours) ${ }^{12} \mathrm{CO}$ emission at medium velocity, high velocity, and very high velocity, respectively. The contour levels start at $4 \sigma$ with a step of $6 \sigma$ in (c), and a step of $4 \sigma$ in $(\mathrm{d})$ and (e), where $\sigma=0.025 \mathrm{Jy}_{\text {beam }}{ }^{-1}$. 


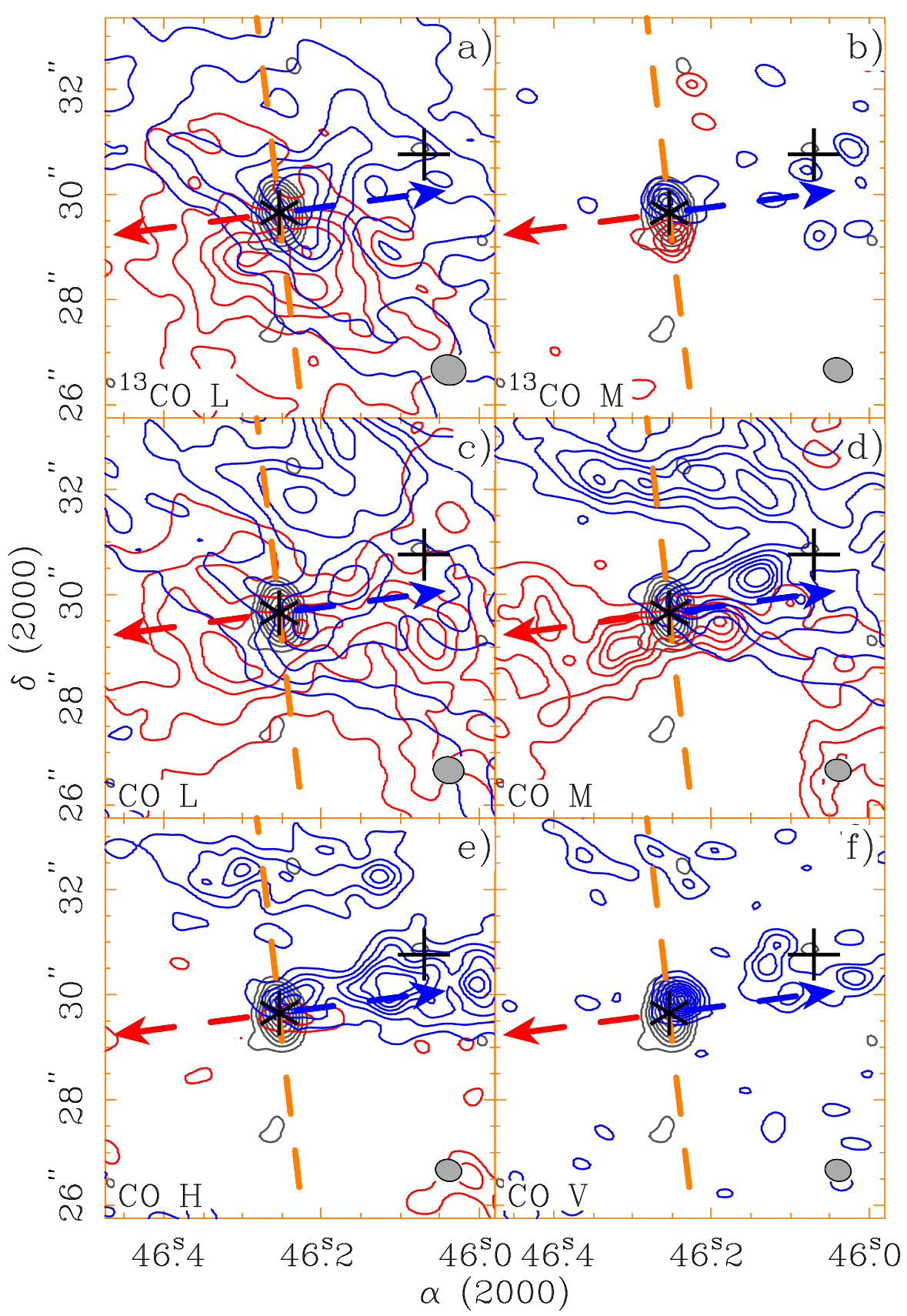

Fig. 2.- Same as Figure 10 but adding low-velocity ${ }^{13} \mathrm{CO}$ and ${ }^{12} \mathrm{CO}$ emission contours, without zooming into the central region. Here (b) corresponds to Figure 1b, and (d)-(f) correspond to Figure 1k-e. (a) shows the the redshifted (red contours) and blueshifted (blue contours) ${ }^{13} \mathrm{CO}$ emission at low velocity. The contour levels start at $4 \sigma$ with a step of $6 \sigma$, where $\sigma=0.023 \mathrm{Jy}$ beam $^{-1}$. (c) shows the the redshifted (red contours) and blueshifted (blue contours) ${ }^{12} \mathrm{CO}$ emission at low velocity. The contour levels start at $4 \sigma$ with a step of $8 \sigma$, where $\sigma=0.025 \mathrm{Jy}^{\text {beam }}{ }^{-1}$. 


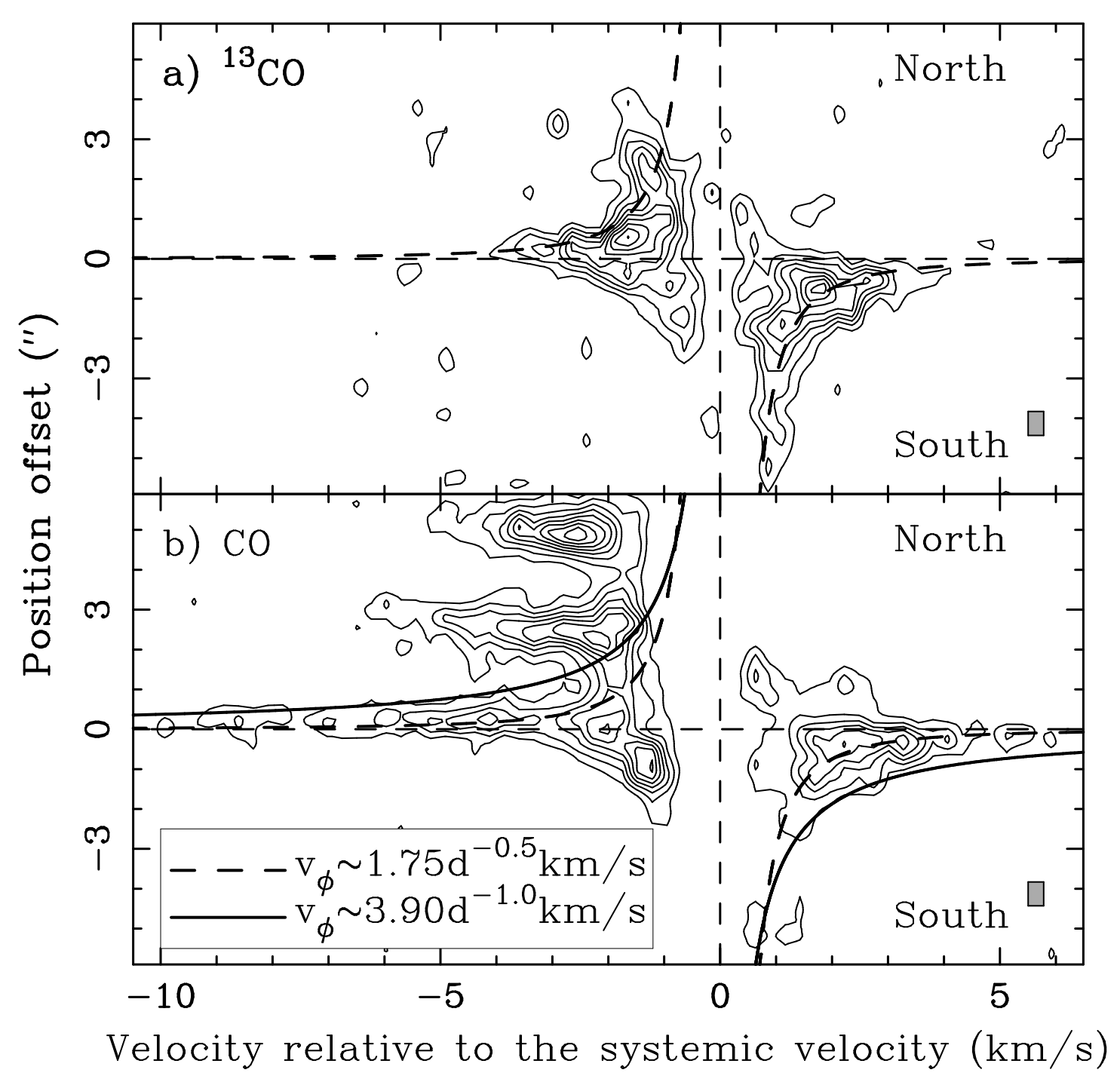

Fig. 3.- Position-velocity (PV) diagrams in (a) ${ }^{13} \mathrm{CO}$ and (b) ${ }^{12} \mathrm{CO}$ centered at the VLA 1 source cut along the equatorial plane perpendicular to the jet axis. The gray box in the lower-right corner shows the velocity and spatial resolutions of the PV diagrams. Solid curve shows the rotation that has constant specific angular momentum and dashed curve shows the Keplerian rotation, as derived from the $\mathrm{C}^{18} \mathrm{O}$ envelope in Paper II, with both corrected for the inclination angle. Here $d$ is the radial distance from the source in arcsec. In (a), the contour levels start at $3 \sigma$ with a step of $3 \sigma$, where $\sigma=0.023 \mathrm{Jy} \mathrm{beam}^{-1}$. In (b), the contour levels start at $3 \sigma$ with a step of $4 \sigma$, where $\sigma=0.025 \mathrm{Jy}^{\text {beam }}{ }^{-1}$. 


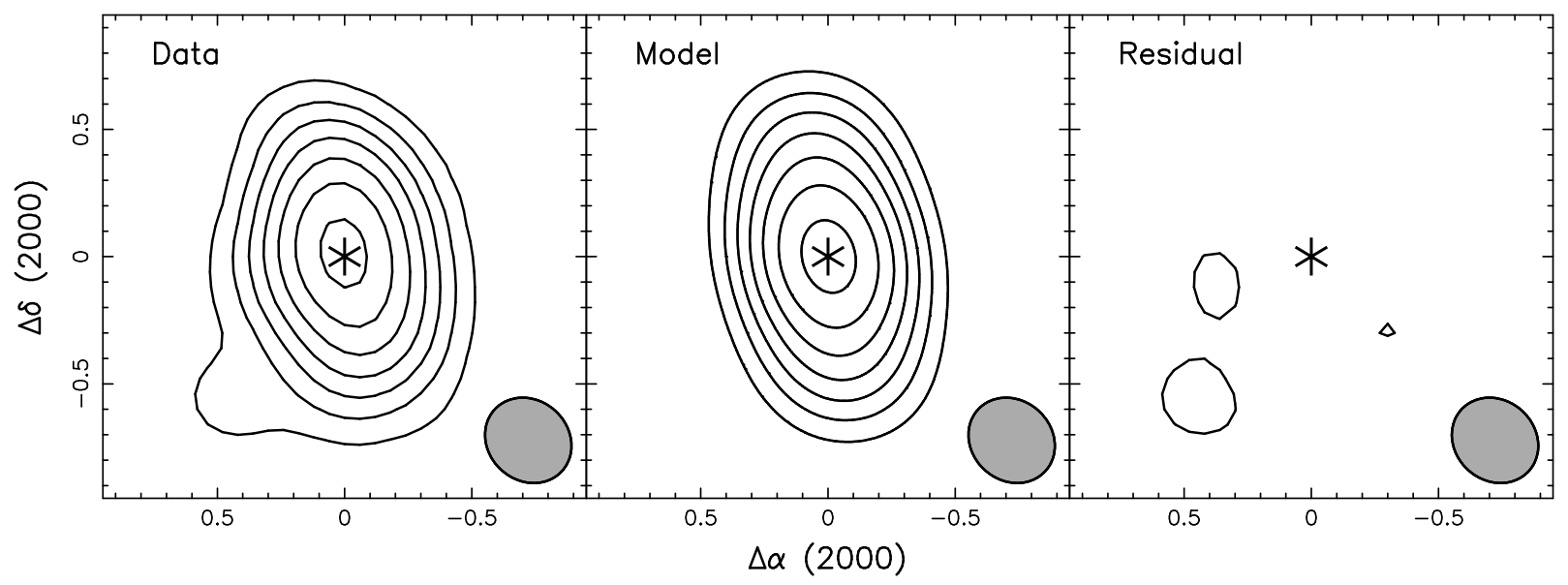

Fig. 4.- A fit to the continuum emission with a simple disk model as described in Section 4. The asterisk marks the position of the VLA 1 source. The contour levels are the same as those in Figure 1a. 


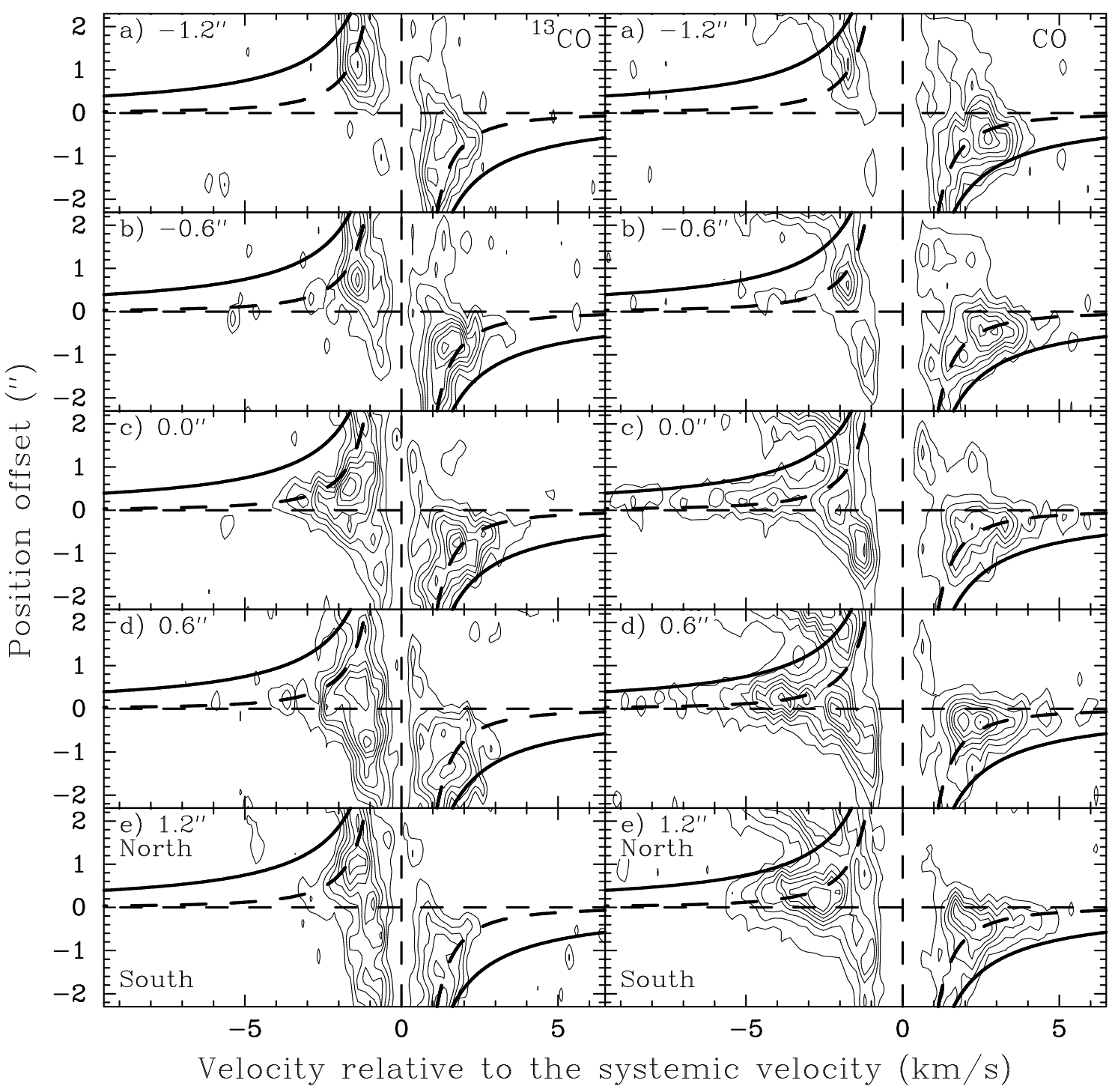

Fig. 5.- PV diagrams in ${ }^{13} \mathrm{CO}$ (left column) and ${ }^{12} \mathrm{CO}$ (right column) cut across the jet axis centered at 5 positions along the jet axis, from (a) -1 ."2 in the east to (e) $1^{\prime \prime} .2$ in the west with a step of 0.6 (which is the size of the synthesized beam). The gray box in the lower-right corner of (a) shows the velocity and spatial resolutions of the PV diagrams. The solid and dashed curves, and contour levels are the same as those in Figure 3 , 